University of Nebraska - Lincoln

DigitalCommons@University of Nebraska - Lincoln

2018

Creation of micro/nano surface structures on silver using

collinear double femtosecond laser pulses with different pulse

separation

Nicholas Roth

Craig Zuhlke

Edwin Peng

Scott Hansen

Jeffrey E. Shield

See next page for additional authors

Follow this and additional works at: https://digitalcommons.unl.edu/nsripubs

Part of the Defense and Security Studies Commons, Emergency and Disaster Management Commons, Other Public Affairs, Public Policy and Public Administration Commons, Science and Technology Policy Commons, and the Terrorism Studies Commons

This Article is brought to you for free and open access by the National Strategic Research Institute at the University of Nebraska at DigitalCommons@University of Nebraska - Lincoln. It has been accepted for inclusion in University of Nebraska's National Strategic Research Institute Publications by an authorized administrator of DigitalCommons@University of Nebraska - Lincoln. 
Authors

Nicholas Roth, Craig Zuhlke, Edwin Peng, Scott Hansen, Jeffrey E. Shield, and Dennis Alexander 


\title{
Creation of micro/nano surface structures on silver using collinear double femtosecond laser pulses with different pulse separation
}

\author{
Nicholas Roth ${ }^{1}$ (I) Craig Zuhlke ${ }^{1} \cdot$ Edwin Peng $^{2} \cdot$ Scott Hansen $^{3} \cdot$ Jeffrey E. Shield ${ }^{2} \cdot$ Dennis Alexander $^{1}$
}

Received: 27 March 2018 / Accepted: 9 April 2018 / Published online: 23 April 2018

c) Springer International Publishing AG, part of Springer Nature 2018

U.S. government works are not subject to copyright.

\begin{abstract}
Self-organized mound-like micro/nanoscale structures are reported for the first time on silver using a dual-pulse femtosecond laser surface processing technique. The dual-pulse laser processing technique reported in this paper uses femtosecond laser pulse pairs with a controlled temporal delay between the leading and trailing pulses. Using dual pulses at higher fluence values, mound-like micro/nanostructures have been created on silver samples for the first time. Formation of the self-organized microstructures is shown to be dependent on the time delay between the leading and trailing pulses. Mound-like microstructures do not develop on silver for overlapped pulses or using single-pulse femtosecond laser surface processing for the parameter space studied. Subsurface microstructure characterization of a single mound-like surface structure is analyzed by crosssectional analysis using focused ion beam milling followed by scanning electron microscopy and energy dispersive X-ray spectroscopy.
\end{abstract}

Keywords Femtosecond $\cdot$ Laser $\cdot$ Silver $\cdot$ Materials $\cdot$ Nanotechnology $\cdot$ Functionalization

\section{Introduction}

Femtosecond laser surface processing (FLSP) is a developing technique for creating micro/nanoscale surfaces with a wide range of applications including improved heat transfer (Kruse et al. 2013, 2015, 2016), medical implants (Vorobyev and Guo 2007), improving efficiency of electrolysis cells (Anderson et al. 2015), and controlling material wetting properties (Zuhlke et al. 2013). In most of these applications, the greatest enhancements result from a surface with a combination of micro and nanoscale surface features. Creation of self-organized micro/nanoscale structures has been demonstrated using FLSP for a range of metals including stainless steel, titanium, nickel, and many other transition metals but

Nicholas Roth

Nicholas.roth@huskers.unl.edu

1 Department of Electrical and Computer Engineering, University of Nebraska-Lincoln, 209N Scott Engineering Center, Lincoln, NE 68588, USA

2 Department of Mechanical and Materials Engineering, University of Nebraska-Lincoln, W342 Nebraska Hall, Lincoln, NE 68588, USA

3 EC6: Thermal Systems Branch, NASA Johnson Space Center, 2101 NASA Parkway, Houston, TX 77058, USA has been challenging in general for noble metals (Vorobyev and Guo 2013). The difficulty in forming microscale surface structures and the difference in structure formation on noble metals versus other metals has not been adequately explained and this paper explores the phenomena.

In this paper, a dual-pulse FLSP technique is used to produce self-organized microscale mound-like structures on silver with controlled temporal delay between the pulse pairs. The mound-like structures are similar to the structures reported in the literature for other metals (Vorobyev and Guo 2007; Kietzig et al. 2009; Kruse et al. 2013; Zuhlke et al. 2013; Peng et al. 2017a,b). The dual-pulse technique reported in this paper uses a similar experimental setup for controlled temporal delay between pulse pairs as pump/probe experiments used in time-resolved spectroscopy. The characteristics of dual-pulse laser interactions have been studied in the past in the context of laser-induced breakdown spectroscopy (LIBS) (Schiffern et al. 2007). Past experiments show that overlapped double pulses result in an increased ablation depth compared to double pulses with a delay greater than $10 \mathrm{ps}$. Therefore, the energy does not go into removal of material when a delay between pulses is introduced (Semerok and Dutouquet 2004). The current consensus, in the literature review, indicates this change in energy absorption for different delay times is a result of plasma shielding from the first 
pulse to interact with the sample surface (Babushok et al. 2006). For the double-pulse technique it has been demonstrated that the ablation threshold energy for the second laser pulse is reduced because of changes in the optical properties of the material from the initial pulse (Bonse et al. 2002).

In this paper, it is reported for the first time that using dual-pulses at higher fluence values, mound-like micro/nanostructures can be created on silver. Dual-pulse FLSP allows for modifying the surface properties of silver through the creation of micro/nanoscale surface features without changing the bulk characteristics.

\section{Experimental}

A schematic of the dual-pulse FLSP experimental setup is shown in Fig. 1. Single-pulse FLSP experiments were carried out using the same setup shown in Fig. 1 by blocking pulses from either the leading or trailing pulse arm. The femtosecond laser used for this work was a Ti:sapphire laser system (Astrella, Coherent) that produces $35 \mathrm{fs}$ duration pulses, with a wavelength centered at $800 \mathrm{~nm}$, a maximum pulse energy of $6 \mathrm{~mJ}$, and a $1 \mathrm{kHz}$ repetition rate. A fused silica thin film polarizer and half-wave plate were used to split each laser pulse into pulse pairs. After being split, the two pulses traveled different distances and were recombined spatially using a thin film polarizer. Each pulse pair was overlapped spatially when focused on the sample surface. An autocorrelator (MINI Autocorrelator, APE) was used to measure the delay between the leading pulses and trailing pulses. The separation between each pulse was controlled using an Aerotech ABL1000 Air-Bearing linear Stage with an accuracy of $2 \mu \mathrm{m}$. The time delay between pulses was controlled by changing the beam path length of the trailing pulse and keeping the path length for the leading pulse constant.

The laser beam spot size for the leading and trailing pulses was measured using a CCD beam profiler (ThorLabs, BC106N-VIS). The laser pulses were focused onto the sample using a $150 \mathrm{~mm}$ focal length plano-convex lens made of fused silica. Unless otherwise stated, the pulse from the leading pulse arm was focused onto the sample to a $e^{-2}$ spot diameter of $615 \mu \mathrm{m}$ and the trailing pulse $e^{-2}$ spot diameter was $520 \mu \mathrm{m}$. The difference in spot size between the two pulses was due to a slight divergence of the trailing beam introduced by the thin film polarizers. The pulse length for the pulses traveling through the leading pulse path was approximately $50 \mathrm{fs}$, measured at the location of the autocorrelator indicated in Fig. 1. The pulses traveling through the trailing pulse path were approximately $100 \mathrm{fs}$. The difference in pulse duration was due to dispersion introduced by the increased amount of glass the second pulse had to transverse in the beam path. The polarization of the leading and trailing pulses is indicated in Fig. 1. After the pulses were combined to be collinear, the polarization of the leading pulses was rotated $90^{\circ}$ relative to the trailing pulses. The sample was placed on a programmable three-dimensional translation stage system used for raster scanning large areas. For all raster scanned samples reported in this paper, the translation velocity of the sample was $4 \mathrm{~mm} / \mathrm{s}$ and the spacing between each raster line was $30 \mu \mathrm{m}$.

Two types of surface processing studies were conducted: ablating a single spot while keeping the sample stationary and raster scanning the sample to process a large area. The silver samples used were $0.8 \mathrm{~mm}$ thick with $99.9 \%$ purity. Prior to laser processing, the silver samples were polished using 1000 grit sandpaper. After polishing, the samples were cleaned in a series of ultrasonic baths of acetone, then methanol, and
Fig. 1 Dual pulse FLSP setup used to conduct this research

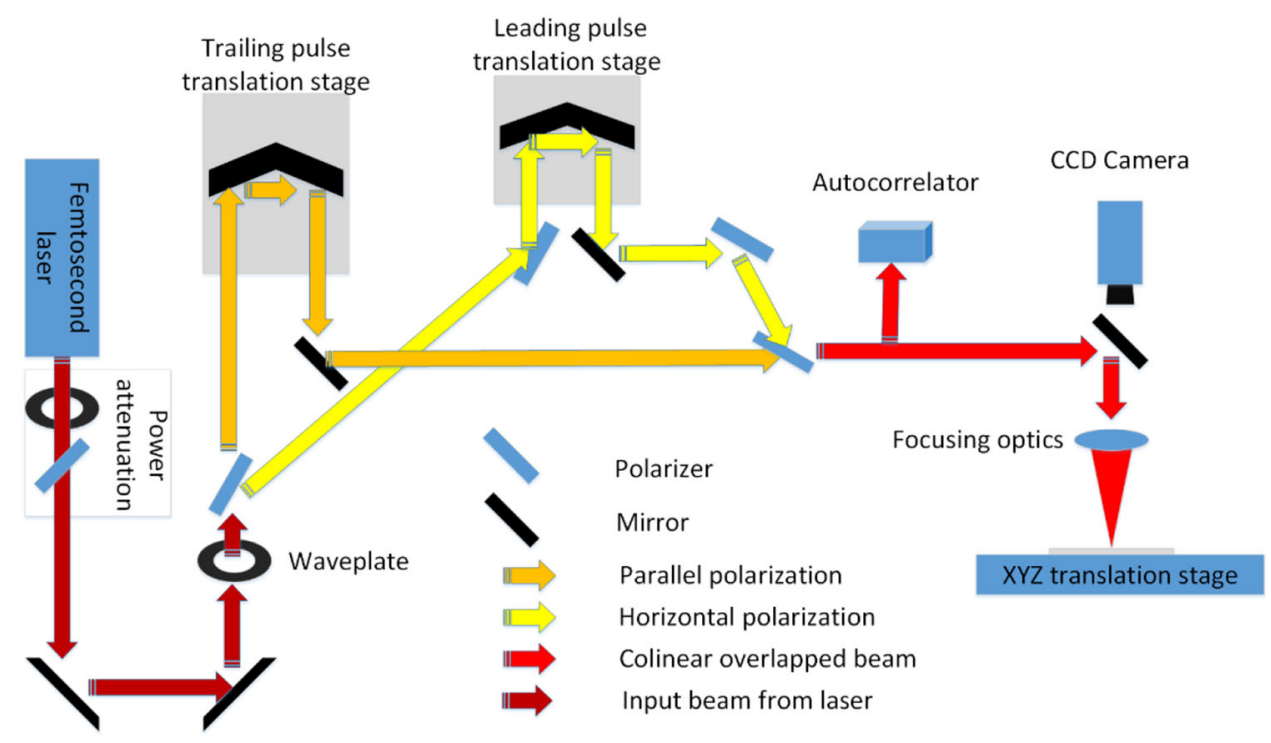



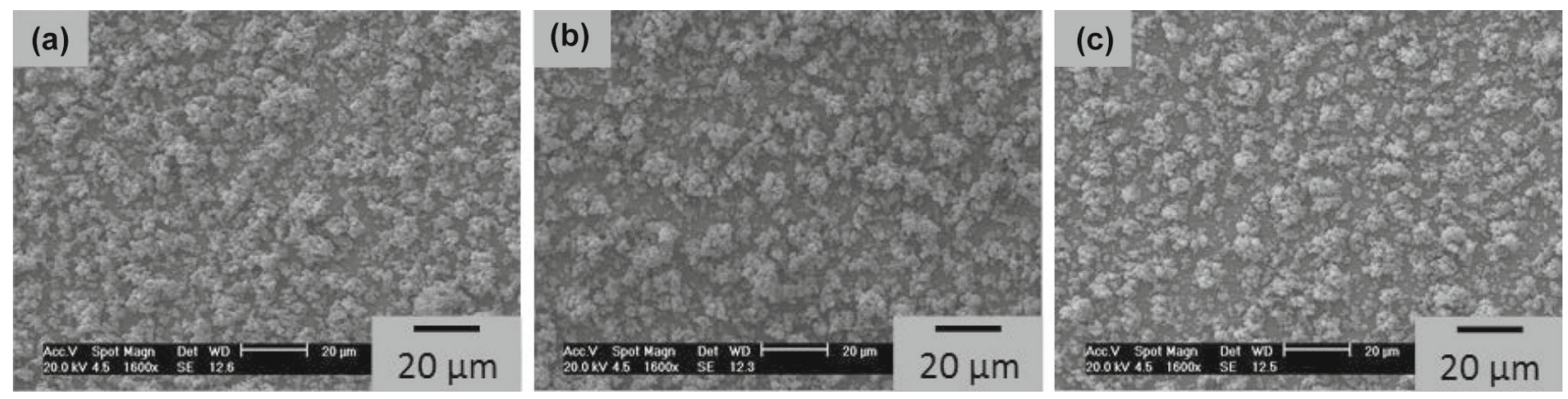

Fig. 2 SEM images of silver using (a) both the overlapped pulses with power split between leading and following pulse (b) full power
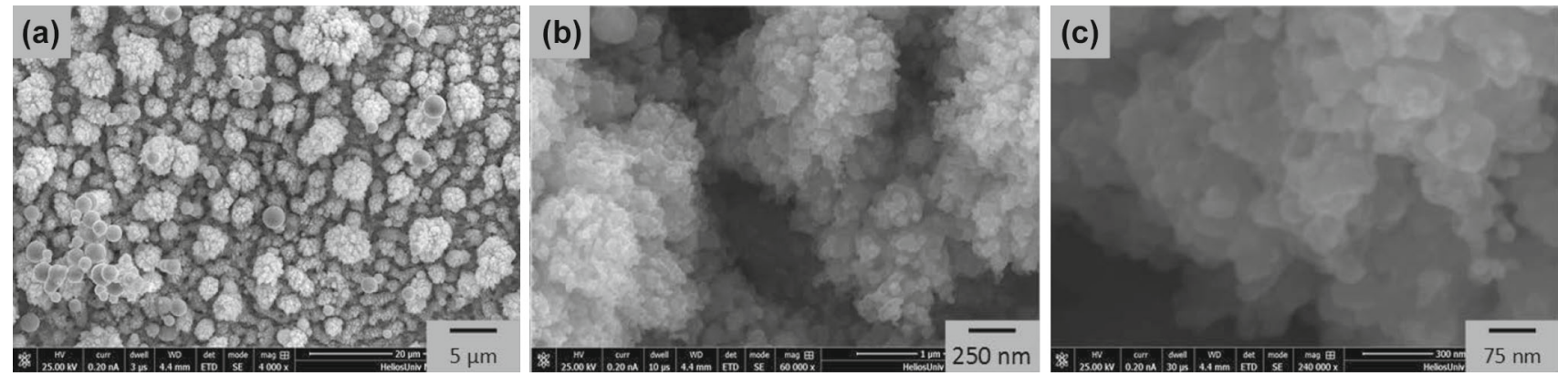

Fig. 3 High resolution SEM image of the nano-particle aggregate found on processed silver after dual pulse FLSP with temporally overlapped pulses. These images were taken of the same sample shown in Fig. 2a

finally distilled water. After laser processing, the samples were characterized using a scanning electron microscope (SEM) and a Keyence VK-X 3D laser scanning confocal microscope (LSCM). The subsurface microstructure of the mound-like structures were analyzed using SEM, focused ion beam (FIB) milling, and energy dispersive X-ray spectroscopy (EDS) in an FEI Helios NanoLab 660 DualBeam system.

A cross-sectioning process was used for analyzing the underlying microstructure of the mound-like structures. Prior to FIB milling, the target mound was protected with platinum $(\mathrm{Pt})$ layers deposited first via electron-beam induced deposition (200 $\mathrm{nm}$ thick), and then by ion beam-induced deposition ( $2 \mu \mathrm{m}$ thick). Then, $\mathrm{Ga}^{+}$FIB milling was used to remove approximately half of the mound, and the exposed subsurface microstructure was imaged using the SEM. Further details on the cross-sectioning process are described in works by Peng (2017) and Peng et al. (2017b).

\section{Results}

\subsection{Single-pulse/overlapped-pulse FLSP studies}

Silver FLSP surfaces formed using single pulses and temporally overlapped dual pulses during raster scanning are displayed in Fig. 2. Figure 2a is an SEM image of a rastered surface with power split evenly between the leading and trail- ing pulses, and with zero time delay between pulses. The SEM images in Fig. 2b, $c$ are of surfaces rastered using only pulses from the leading beam and the trailing beam, respectively. The peak fluence of the leading pulses was $3.08 \mathrm{~J} / \mathrm{cm}^{2}$ while the peak fluence of the trailing pulses was $4.32 \mathrm{~J} / \mathrm{cm}^{2}$. The total pulse energy of either single-pulse illumination or overlapped dual-pulse illumination was $4.6 \mathrm{~mJ}$.

The surface topography created with single-pulse FLSP consisted of a surface covered with what appears to be aggregated nanoparticles similar to structures reported in the past on aluminum, silicon and silicon carbide (Zuhlke et al. 2010; Tsubaki et al. 2017). The nanoscale roughness of the surface in Fig. 2a can be seen in the high resolution SEM images included in Fig. 3. FLSP mound-like surface structures could not be formed on silver with single-pulse illumination or temporally overlapped dual pulses over the parameter space studied. Increasing laser fluence past $20 \mathrm{~J} / \mathrm{cm}^{2}$ and lowering rastering speeds (increasing laser pulse count) below $1 \mathrm{~mm} / \mathrm{s}$ did not result in the formation of any microscale moundlike surface features. At these illumination conditions, the bulk material was removed and a micro/nano-particle layer formed similar to the surfaces shown in Fig. 2.

\subsection{Stationary double-pulse FLSP studies}

SEM images of stationary ablation spots on silver for different delay times between the leading and trailing pulses are included in Fig. 4. The effect of the delay between the lead- 


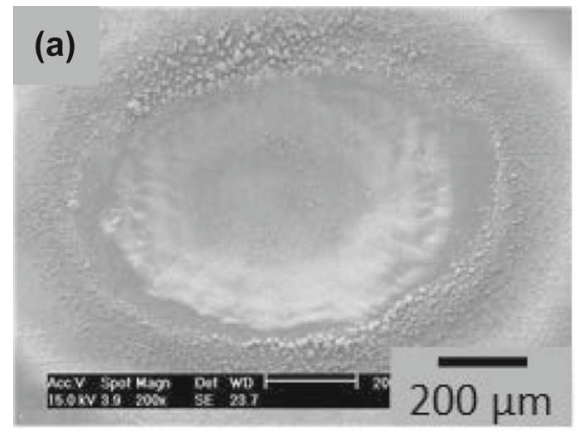

Delay: 0 ps

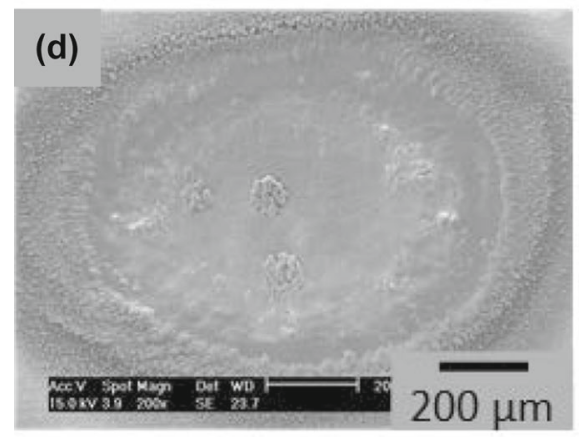

Delay: 80 ps

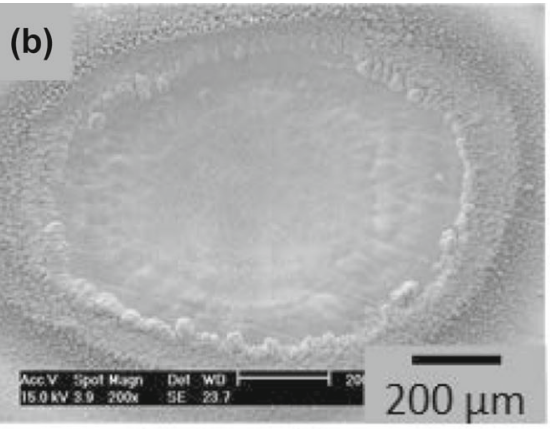

Delay: 40 ps

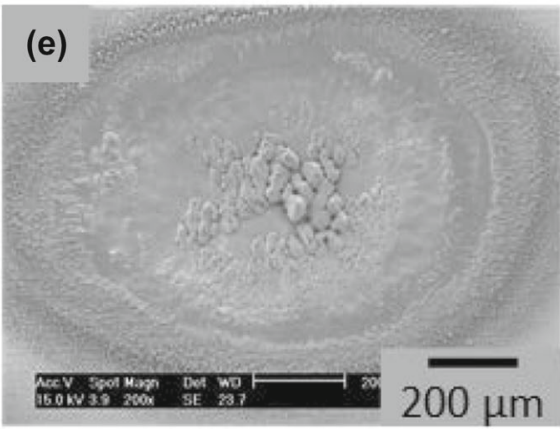

Delay: 95 ps

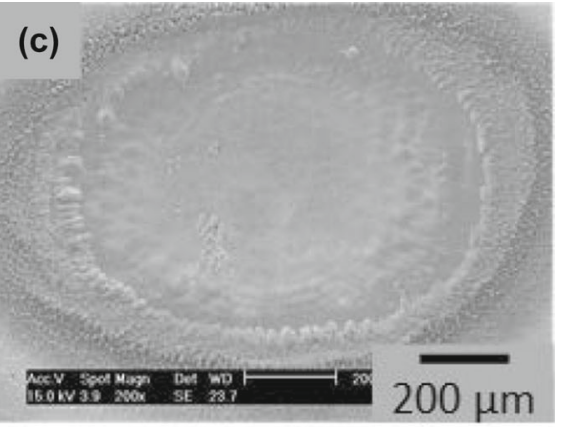

Delay: 65 ps

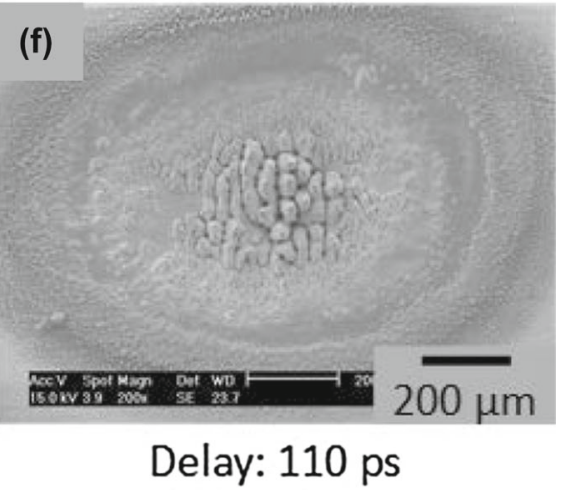

Fig. 4 SEM images of stationary ablation spots in relation to delay between leading and trailing pulse with 3000 pulse pairs at $1.55 \mathrm{~J} / \mathrm{cm}^{2}$ leading pulse peak fluence and $2.17 \mathrm{~J} / \mathrm{cm}^{2}$ trailing pulse peak fluence

ing and trailing laser pulses was studied with the fluence and pulse count being held constant. These ablation spots were made using 3000 pulse pairs with the leading pulses having a peak fluence of $1.55 \mathrm{~J} / \mathrm{cm}^{2}$ and the trailing pulses having a peak fluence of $2.17 \mathrm{~J} / \mathrm{cm}^{2}$.

For stationary dual-pulse FLSP on silver the onset for initial creation of mound-like surface structures occurred at a time delay of $80 \mathrm{ps}$ for the laser parameters used. Increasing the delay to $95 \mathrm{ps}$ and subsequently to $110 \mathrm{ps}$ resulted in progressively more developed structures or structures covering a larger portion of the ablation crater. Delay times past 110 ps were studied but the ablation craters appeared similar for delays between 110 and 300 ps. At delays past 300 ps the surface structures started to disappear.

\subsection{Double-pulse FLSP studies for rastered samples}

For applications of FLSP, it is important to be able to produce micro/nanostructures over a large area on a surface. To get surface modifications that are uniform and repeatable all areas must be exposed to the same laser parameters and pulse counts. This is achieved using a programmable xyz stage system and moving the sample in a raster scanning pattern with a set raster velocity and distance between raster lines. This section investigates the structures created through this raster scanning process with different temporal delays between pulses.

Scanning electron microscope images of surfaces processed using dual-pulse FLSP raster scanning with different dual-pulse temporal delays is included in Fig. 5. The height of the surface structures for the surfaces shown in Fig. 5 was measured using the LSCM. Figure 6 is a graph of the average structure height for the surfaces in Fig. 5. Little difference in structure height was found between single-pulse rastering and dual-pulse rastering until a temporal delay of 80 and 120 ps.

\subsection{Subsurface analysis of a dual-pulse silver mound}

To gain insight into the formation processes of the dualpulse FLSP mound-like structures on silver, the sub-surface microstructure was analyzed using SEM-FIB cross-sectional milling. The mound that was analyzed using cross-sectional analysis was produced using stationary ablation with a temporal pulse spacing of $168 \mathrm{ps}$, a peak fluence of $0.36 \mathrm{~J} / \mathrm{cm}^{2}$ for the leading pulse, a peak fluence of $0.45 \mathrm{~J} / \mathrm{cm}^{2}$ for the trailing pulse and 500 pulse pairs. Figure $7 \mathrm{a}$ includes an SEM image of the ablation crater used in the cross-sectional analysis. Figure $7 \mathrm{~b}$ is a higher magnification SEM image of a single 

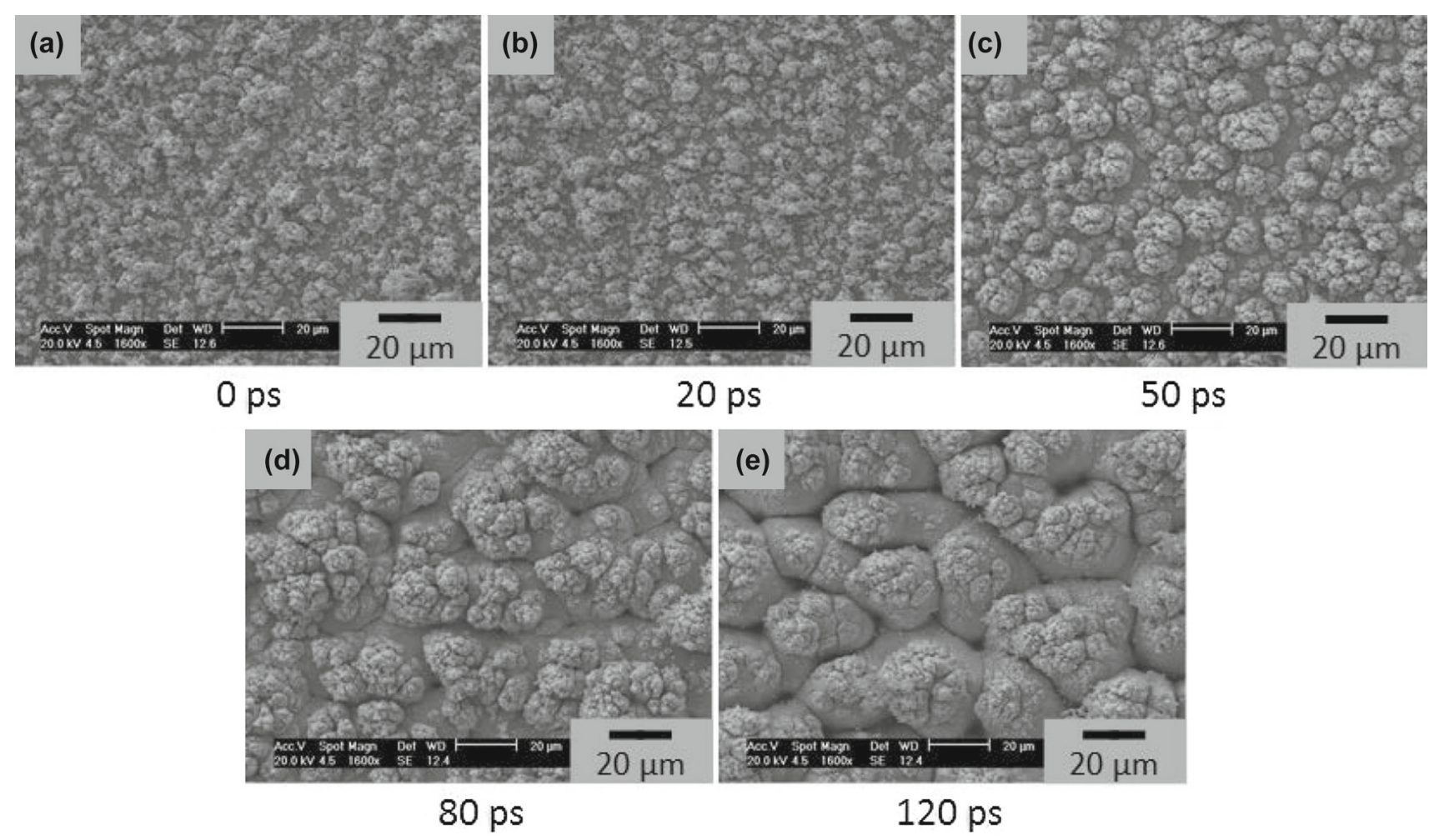

20 ps

50 ps

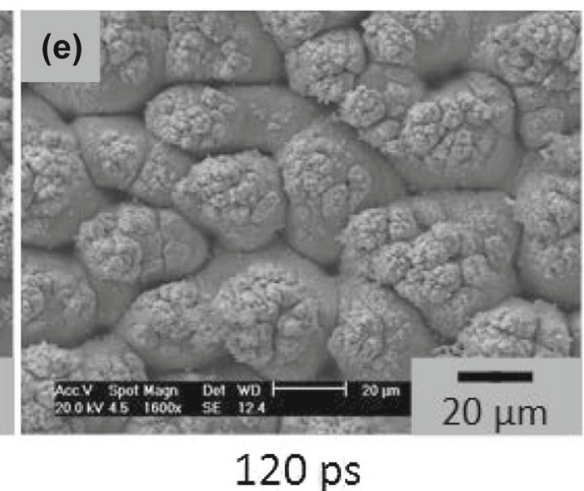

Fig. 5 SEM images of rastered silver surfaces at $\times 1600$ magnification with the delay between the leading and trailing pulse given in picoseconds. The laser fluence used was kept the same as that used for the

stationary ablation spots in Fig. 4. The raster velocity was $4 \mathrm{~mm} / \mathrm{s}$ and the spacing between raster scans was 30 microns

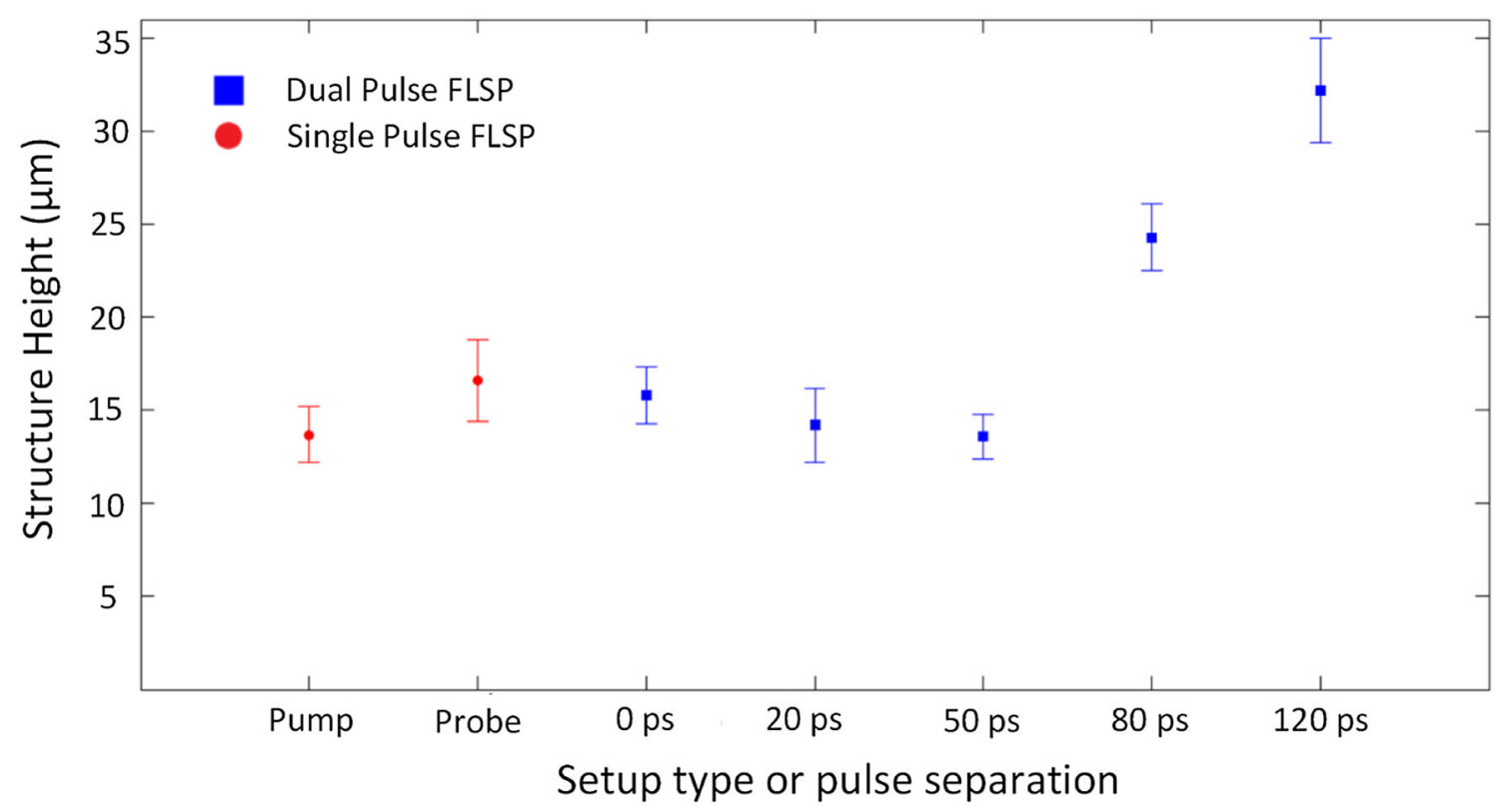

Fig. 6 Average structure height and standard error of surfaces shown in Fig. 5 
Fig. 7 a SEM image of the ablation crater on silver with self-organized mound structures prior to FIB mill cross-sectioning. b SEM image of the mound used in the cross-sectional analysis prior to FIB mill cross-sectioning. c SEM image of the mound after FIB mill cross-sectioning
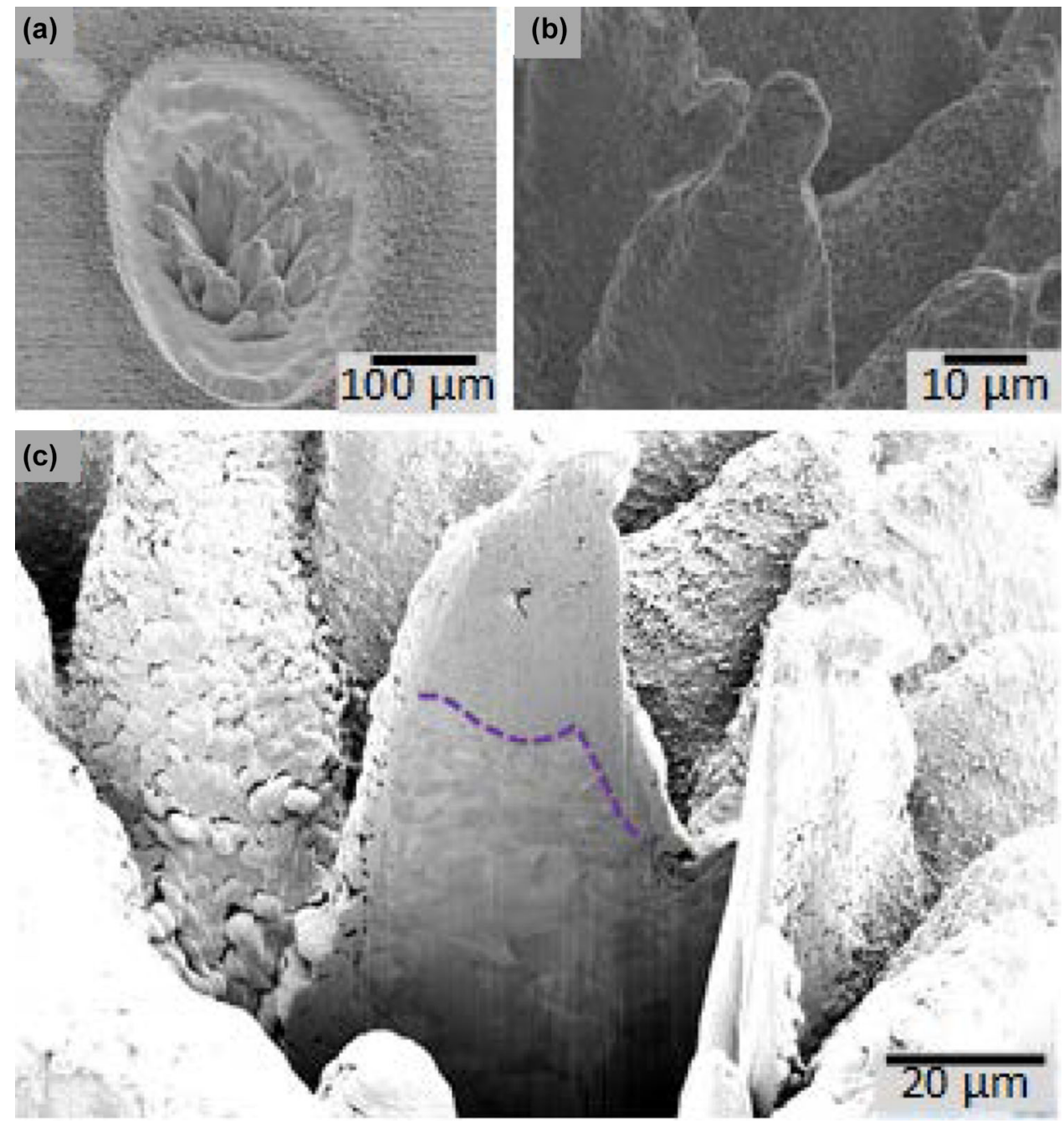

mound prior to cross-sectioning and Fig. 7c is an SEM image of the mound after cross-sectioning with the FIB mill.

Through the cross-sectional analysis, it was found that the bottom half of the mound consists of grains that are similar to the original non-laser-processed material, while the top half consisted of much smaller grains with a large number of voids. The voids show up as black regions in the SEM images. The voids are visible in Fig. 7c but are more easily identified in the higher magnification SEM image included in Fig. 8d. The dashed line in Fig. 7c marks the transition between the larger original grains near the bottom of the structure and the much smaller grains in the top half of the structure. Figure $8 \mathrm{a}$ includes a cross-sectional SEM image showing the original grain sizes from an unprocessed portion of the silver material. The microstructure consists of grains with a size of around $5 \mu \mathrm{m}$. Figure $8 \mathrm{~b}$ is a magnified view of the transition from larger grains to the region with smaller grains and voids. Figure 8c, d are magnified views of the lower portion of the mound and upper portion of the mound. The grains in the lower portion of the mound, visible in Fig. 8c, are much similar in size than the grains in the original material shown in Fig. 8a. The microstructure in the top portion of the mound consists of submicron grain sizes with microscale voids throughout. The vertical lines evident in Fig. 8a-c are artifacts of the ion beam milling process.

\section{Discussion}

The lack of surface structure formation while performing single-pulse FLSP on silver has several possible explanations based on the findings in this paper and the related literature on laser-matter interactions. One possible explanation is related to the electron-phonon coupling constant of silver. When a femtosecond laser pulse interacts with a material energy is first transferred to the electrons within the material. The two-temperature model (TTM) is used throughout the literature to explain the transfer of energy from the hot electrons to vibrations within the material lattice by phonon energy transport. In the TTM model, the transfer of energy from the 
(a)
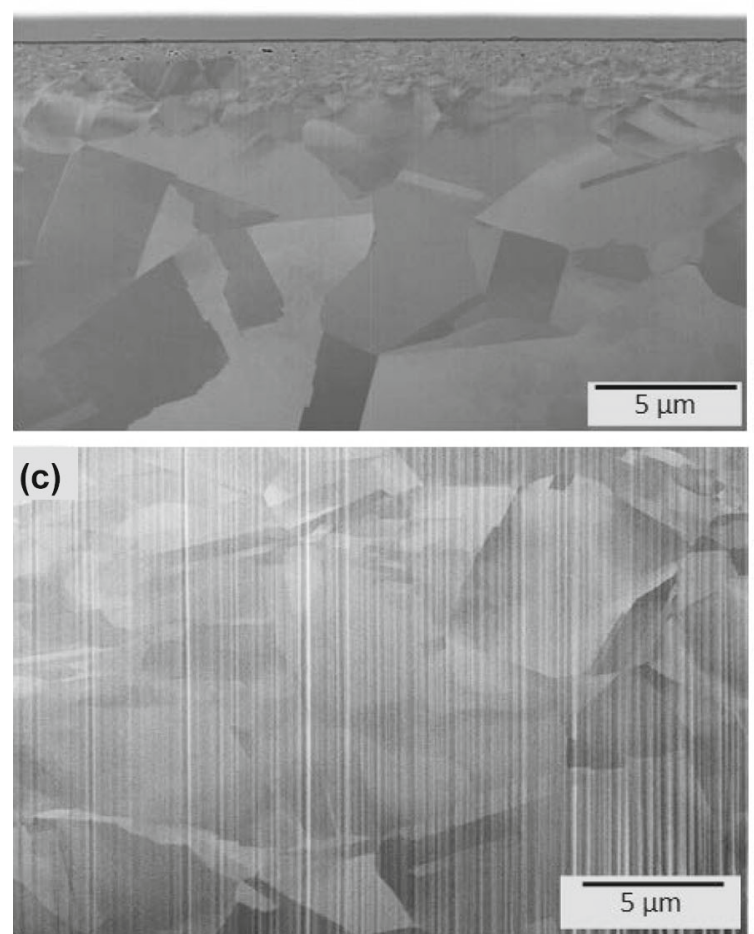

Fig. 8 a SEM images of subsurface grain structure on unprocessed silver showing larger Ag grains below a smaller-grained surface (smaller grains are a result of polishing). b SEM image of the interface between the bottom portion of the mound with the original sized silver grains

hot electrons to the relatively cold lattice is governed by the electron-phonon (el-ph) coupling constant (Chichkov et al. 1996; Wellershoff et al. 1999). Values for the el-ph coupling constant at room temperature are given in Table 1 along with whether or not mound-like micro/nano-structures are created through single-pulse FLSP. The value of the el-ph coupling constant is an order of magnitude lower for silver when compared to nickel, stainless steel, or titanium. The implication of a low coupling constant is a slower transfer of energy from electrons to phonons within the lattice; the energy stored within the electrons disperses further into the bulk material and any laser-induced effects are spread out over a larger region (Tanvir Ahmmed et al. 2014).

The formation of structures through dual-pulse FLSP can be explained by the temperature dependence of the coupling constant $g$. Based on computational analysis published by Lin et al. (2008), an increase in the electron temperature of silver will cause the value of the e-ph coupling constant to increase. Thus, suggesting that the initial pulse heats up the surface/electrons and the trailing pulse encounters a completely different set of material properties compared to the initial pulse (Lin et al. 2008)
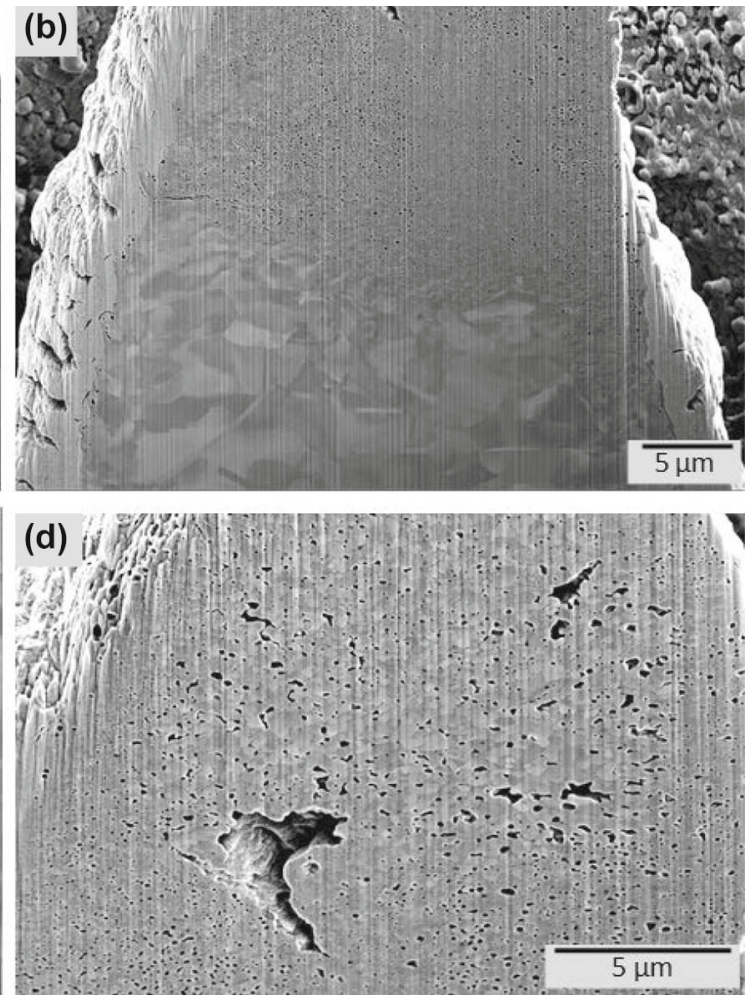

and the top portion of the mound with smaller grain sizes and voids. $\mathbf{c}$ SEM image of the bottom portion of the mound with the original sized silver grains. d SEM image of the top portion of the mound with small grain sizes and voids

Another possible explanation for why surface structures do not form on silver using single-pulse FLSP but do using dual-pulse FLSP is because of laser-induced plasma dynamics. The highly reflective nature of laser-induced plasmas has been studied widely (von der Linde and Schüler 1995; Babushok et al. 2006; Mishra et al. 2017). The creation of a laser-induced plasma forms within the same time-frame as the pulse length. This implies that the trailing edge of a laser pulse will see different surface/plasma conditions than the leading part of a laser pulse because of the formation of a highly dense reflective plasma (Penczak et al. 2014). With the dual-pulse technique, the power is split between two pulses, therefore, the plasma formation from each individual pulse is significantly less than for a single pulse with twice the energy. If the temporal spacing is greater than tens of picoseconds there will be significant decay in the electron density of the plasma before the second pulse arrives (Chizhov et al. 2015), which will reduce any plasma shielding. As an alternative explanation, when using the dual-pulse technique the second pulse may reheat the plasma from the first pulse, resulting in a hotter plasma than when using single-pulse FLSP (Zhao and Shin 2015). The hotter plasma may be needed to drive 
Table 1 Electron-phonon coupling constants and whether formation of FLSP structures occurs for various materials

\begin{tabular}{lll}
\hline & E-P coupling constant $\left(10^{17} \mathrm{~W} \mathrm{~m}^{-3} \mathrm{~K}^{-1}\right)$ & Single pulse FLSP structure formation \\
\hline Silver & 0.35 (Groeneveld et al. 1995) & None \\
Nickel & 8 (Beaurepaire et al. 1996) & Yes (Zuhlke et al. 2013) \\
Titanium & 18.5 (Ahmmed et al. 2015) & Yes (Vorobyev and Guo 2007) \\
304 Stainless steel & 14.4 (Winter et al. 2016) & Yes (Kruse et al. 2015) \\
\hline
\end{tabular}

the self-organization processes for the structures to form on silver.

Based on the subsurface microstructure analysis the formation of the mounds is likely a balance between preferential ablation and fluid-flow or redeposition of ablated material. The subsurface microstructure, with a balance between original grains sizes at the base of the structure and smaller grain sizes near the surface is similar to what has been observed through single-pulse FLSP on other materials (Peng et al. 2016, 2017a,b). These similarities indicate similar formation processes for the dual-pulse structures on silver as single pulse structures on other materials. The presence of the original grains in the bottom portion of the structure indicates that material was selectively removed from the valleys around the structure through preferential ablation processes (Zuhlke et al. 2013). Due to the minimized heat affected zone associated with femtosecond ablation the subsurface microstructure would remain unaltered during the preferential ablation process (Zuhlke et al. 2013). The small grain sizes and large number of voids in the upper portion of the mound structure indicate either fluid flow of the surface melt induced by the laser pulses or redeposition of ablated material on top of the structure resulted in upward growth of the structure similar to what has been reported in previous work on nickel (Zuhlke et al. 2013), polycrystalline Ni60Nb40 (Peng et al. 2016, 2017b) and titanium (Peng et al. 2017a). While voids in the subsurface microstructure have been previously observed for single-pulse FLSP of titanium (Peng et al. 2017a), the dual-pulse silver mound structure contains a higher density of voids. Further research is needed to determine if the increased density of voids is related to the dual-pulse technique or is related to a property of silver.

\section{Conclusion}

Mound-like micro/nanostructures were successfully created on silver using dual-pulse femtosecond laser surface processing (FLSP). The influence of temporal spacing between leading and trailing pulses was investigated and a pulse separation greater than $80 \mathrm{ps}$ is needed to induce structure formation for the processing parameters used. Mound-like microstructures were created on silver using both station- ary ablation spots and rastered surface areas. The structures created within the stationary ablation craters were found to be similar in composition to past structures created on other materials using single-pulse FLSP. There are a number of possible explanations for why dual-pulse FLSP results in the creation of mound-like structures on silver, while singlepulse FLSP does not. Further work is needed to understand the physics of dual-pulse FLSP.

Acknowledgements This work was supported by the Office of Naval Research (ONR) Contract number FA4600-12-D-9000-0057, NASA Johnson Space Center Contract number NNJ17HH85P and the NASA Space Grant Consortium FY 2015-FY 2017 Grant \# NNX15AI09H.

The research was performed in part in the Nebraska Nanoscale Facility: National Nanotechnology Coordinated Infrastructure and the Nebraska Center for Materials and Nanoscience, which are supported by the National Science Foundation under Award ECCS: 1542182, and the Nebraska Research Initiative.

\section{References}

Ahmmed KMT, Ling EJY, Servio P, Kietzig AM (2015) Introducing a new optimization tool for femtosecond laser-induced surface texturing on titanium, stainless steel, aluminum and copper. Opt Lasers Eng 66:258-268. https://doi.org/10.1016/j.optlaseng.2014. 09.017

Anderson TP, Wilson C, Zuhlke CA et al (2015) Enhancing vapor generation at a liquid-solid interface using micro/nanoscale surface structures fabricated by femtosecond laser surface processing. Proc SPIE 9351:9351. https://doi.org/10.1117/12.2079828

Babushok VI, DeLucia FC, Gottfried JL et al (2006) Double pulse laser ablation and plasma: laser induced breakdown spectroscopy signal enhancement. Spectrochim Acta Part B At Spectrosc 61:999 1014. https://doi.org/10.1016/j.sab.2006.09.003

Beaurepaire E, Merle JC, Daunois A, Bigot JY (1996) Ultrafast spin dynamics in ferromagnetic nickel. Phys Rev Lett 76:4250-4253. https://doi.org/10.1103/PhysRevLett.76.4250

Bonse J, Wrobel JM, Brzezinka KW et al (2002) Femtosecond laser irradiation of indium phosphide in air: Raman spectroscopic and atomic force microscopic investigations. Appl Surf Sci 202:272282. https://doi.org/10.1016/S0169-4332(02)00948-0

Chichkov BN, Momma C, Nolte S et al (1996) Femtosecond, picosecond and nanosecond laser ablation of solids. Appl Phys A Mater Sci Process 63:109-115. https://doi.org/10.1007/s003390050359

Chizhov P, Bukin V, Garnov S (2015) Interferometry in femtosecond laser plasma diagnostics. Phys Proc 71:222-226. https://doi.org/ 10.1016/j.phpro.2015.08.363

Groeneveld RHM, Sprik R, Lagendijk A (1995) Femtosecond spectroscopy of electron-electron and alectron-phonon energy relax- 
ation in Ag aund Au. Phys Rev B 51:11433. https://doi.org/10. 1103/PhysRevB.51.11433

Kietzig A-M, Hatzikiriakos SG, Englezos P (2009) Patterned superhydrophobic metallic surfaces. Langmuir 25:4821-7. https://doi.org/ $10.1021 / 1 \mathrm{a} 8037582$

Kruse C, Anderson T, Wilson C et al (2013) Extraordinary shifts of the leidenfrost temperature from multiscale micro/nanostructured surfaces. Langmuir 29(31):9798-9806. https://doi.org/10.1021/ la401936w

Kruse C, Tsubaki A, Zuhlke C et al (2016) Secondary pool boiling effects. Appl Phys Lett 108:51602. https://doi.org/10.1063/ 1.4941081

Kruse CM, Anderson T, Wilson C et al (2015) Enhanced pool-boiling heat transfer and critical heat flux on femtosecond laser processed stainless steel surfaces. Int J Heat Mass Transf 82:109-116. https:// doi.org/10.1016/j.ijheatmasstransfer.2014.11.023

Lin Z, Zhigilei LV, Celli V (2008) Electron-phonon coupling and electron heat capacity of metals under conditions of strong electronphonon nonequilibrium. Phys Rev B Condens Matter Mater Phys 77:1-17. https://doi.org/10.1103/PhysRevB.77.075133

Mishra SK, Andreev A, Kalashinikov MP (2017) Reflection of few cycle laser pulses from an inhomogeneous overdense plasma. Opt Express 25:11637. https://doi.org/10.1364/OE.25.011637

Penczak J, Kupfer R, Bar I, Gordon RJ (2014) The role of plasma shielding in collinear double-pulse femtosecond laser-induced breakdown spectroscopy. Spectrochim Acta Part B At Spectrosc 97:34-41. https://doi.org/10.1016/j.sab.2014.04.007

Peng E (2017) Understanding the formation of micro/nanoscale structures on metal surfaces by ultrafast pulse laser processing. ProQuest Dissertations and Theses. ISBN:9780355506266. https:// search.proquest.com/docview/1975743172?accountid=8116

Peng E, Bell R, Zuhlke CA et al (2017a) Growth mechanisms of multiscale, mound-like surface structures on titanium by femtosecond laser processing. J Appl Phys 122:133108. https://doi.org/10. 1063/1.4990709

Peng E, Tsubaki A, Zuhlke CA et al (2017b) Micro/nanostructures formation by femtosecond laser surface processing on amorphous and polycrystalline Ni60Nb40. Appl Surf Sci 396:1170-1176. https:// doi.org/10.1016/j.apsusc.2016.11.107

Peng E, Tsubaki A, Zuhlke CA et al (2016) Experimental explanation of the formation mechanism of surface mound-structures by femtosecond laser on polycrystalline Ni60Nb40. Appl Phys Lett 108:31602. https://doi.org/10.1063/1.4939983
Schiffern JT, Doerr DW, Alexander DR (2007) Optimization of collinear double-pulse femtosecond laser-induced breakdown spectroscopy of silicon. Spectrochim Acta Part B At Spectrosc 62:1412-1418. https://doi.org/10.1016/j.sab.2007.10.042

Semerok A, Dutouquet C (2004) Ultrashort double pulse laser ablation of metals. Thin Solid Films 453-454:501-505. https://doi.org/10. 1016/j.tsf.2003.11.115

Tanvir Ahmmed KM, Grambow C, Kietzig AM (2014) Fabrication of micro/nano structures on metals by femtosecond laser micromachining. Micromachines 5:1219-1253. https://doi.org/10.3390/ mi5041219

Tsubaki AT, Koten MA, Lucis MJ et al (2017) Formation of aggregated nanoparticle spheres through femtosecond laser surface processing. Appl Surf Sci 419:778-787. https://doi.org/10.1016/j.apsusc. 2017.05.094

von der Linde D, Schüler H (1995) Observation of the dynamics of electron plasma oscillations in femtosecond laser-produced plasmas. Appl Phys Lett 66:807. https://doi.org/10.1063/1.113427

Vorobyev a Y, Guo C (2007) Femtosecond laser structuring of titanium implants. Appl Surf Sci 253:7272-7280. https://doi.org/10.1016/ j.apsusc.2007.03.006

Vorobyev AY, Guo C (2013) Direct femtosecond laser surface nano/microstructuring and its applications. Laser Photon Rev 7:385-407. https://doi.org/10.1002/lpor.201200017

Wellershoff S-S, Hohlfeld J, Güdde J, Matthias E (1999) The role of electron-phonon coupling in femtosecond laser damage of metals. Appl Phys A 69(1):S99-S107. https://doi.org/10.1007/ s003399900305

Winter J, Sotrop J, Borek S et al (2016) Temperature-dependent determination of electron heat capacity and electron-phonon coupling factor for Fe0.72Cr0.18Ni0.1. Phys Rev B 93:1-8. https://doi.org/ 10.1103/PhysRevB.93.165119

Zhao X, Shin YC (2015) Laser-plasma interaction and plasma enhancement by ultrashort double-pulse ablation. Appl Phys B Lasers Opt 120:81-87. https://doi.org/10.1007/s00340-015-6102-4

Zuhlke CA, Alexander DR, Bruce JC et al (2010) Self assembled nanoparticle aggregates from line focused femtosecond laser ablation. Opt Express 18:4329-39

Zuhlke CA, Anderson TP, Alexander DR (2013) Formation of multiscale surface structures on nickel via above surface growth and below surface growth mechanisms using femtosecond laser pulses. Opt Express 21:8460. https://doi.org/10.1364/OE.21.008460

Publisher's Note Springer Nature remains neutral with regard to jurisdictional claims in published maps and institutional affiliations. 